\title{
The spectrum of persistence of testicular blastema and ectopic testicular parenchyma: a possible result of focal delay in gonadal development
}

Mariana M. Cajaiba • Eugenia García-Fernández • Miguel Reyes-Múgica $\cdot$ Manuel Nistal

Published online: 3 August 2007

(C) Springer-Verlag 2007

Unfortunately the addresses were published with errors.

The correct addresses are given below.

The online version of the original article can be found at DOI:10.1007/s00428-007-0405-8.

M. M. Cajaiba • E. García-Fernández • M. Reyes-Múgica •

M. Nistal

Department of Pathology, Hospital La Paz/

Universidad Autónoma de Madrid,

Madrid, Spain

M. M. Cajaiba $(\square) \cdot$ M. Reyes-Múgica

Program of Pediatric and Developmental Pathology,

Yale University School of Medicine,

430 Congress Avenue,

New Haven, CT 06520, USA

e-mail: mmcajaiba@yahoo.com 